Supplement of Atmos. Chem. Phys. Discuss., 15, 31501-31536, 2015

http://www.atmos-chem-phys-discuss.net/15/31501/2015/

doi:10.5194/acpd-15-31501-2015-supplement

(C) Author(s) 2015. CC Attribution 3.0 License.

(c) (i)

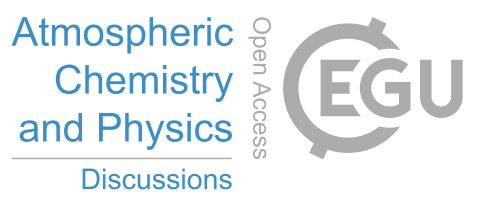

Supplement of

\title{
In situ measurements and modeling of reactive trace gases in a small biomass burning plume
}

M. Müller et al.

Correspondence to: A. Wisthaler (armin.wisthaler@uibk.ac.at)

The copyright of individual parts of the supplement might differ from the CC-BY 3.0 licence. 


\section{Slow instrument response for "sticky" analytes}

The PTR-ToF-MS instrument has a response time of $\sim 0.1 \mathrm{~s}$. Signal rise and fall times may, however, increase to seconds or even tens of seconds for "sticky" analytes such as acetic acid (Figure S1).

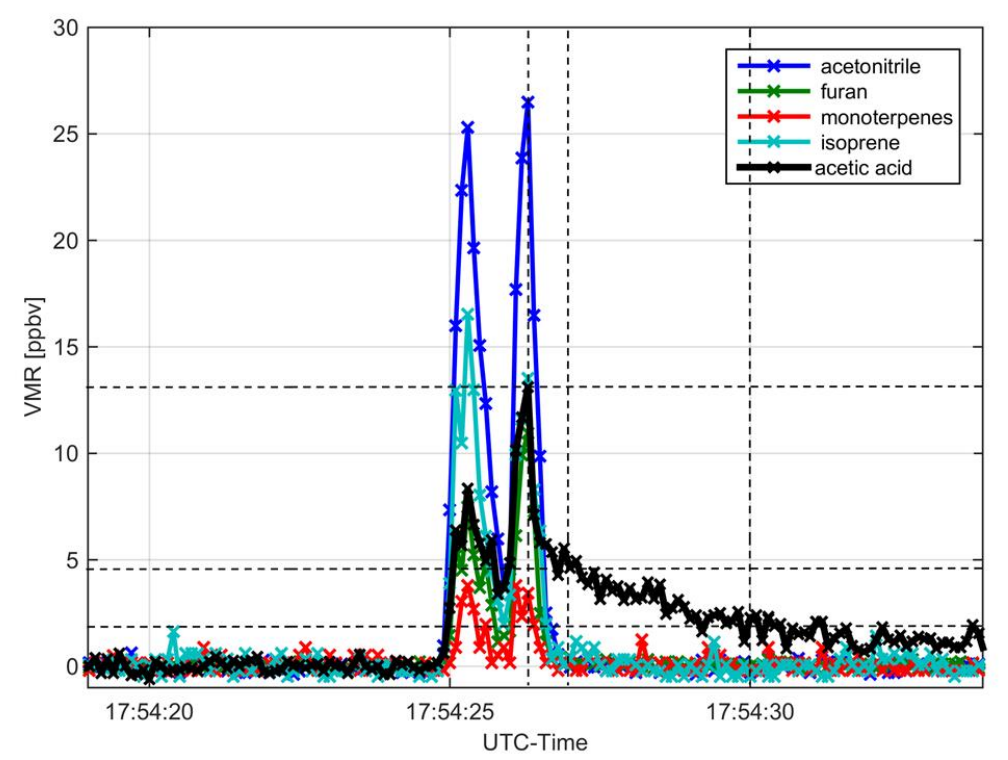

Figure S1. $10 \mathrm{~Hz}$ time-series of (a) acetonitrile, furan, the sum of monoterpene isomers, isoprene and acetic acid as measured during the fourth fire overflight at 17:54:25 UTC.

During the plume encounter, acetic acid molecules are partly adsorbed onto instrumental surfaces which results in an underestimation of in-plume concentrations. After the plume encounter, surface-bound molecules desorb from instrumental surfaces causing the associated signal to slowly decrease to atmospheric background values. Our experience from laboratory experiments indicates that the fraction of adsorbed analyte molecules is quantitatively recovered during desorption. A cumulative signal analysis which includes the period after the plume encounter will thus correctly quantify the amount of analyte in the plume. Figure S2 shows the cumulative mixing ratios of acetonitrile, formic acid and acetic acid as observed during the second fire overflight. The instrument response to acetonitrile is instantaneous whereas significant delays are observed for the two acids. All other signals reached $90 \%$ of their cumulative mixing ratios within the initial $10 \mathrm{~s}$ after the plume encounter. Only in the case of formic acid and acetic acid we thus used a cumulative signal analysis for emission quantification. In the case of formic acid we only report upper limit values due to a poor detection limit. 


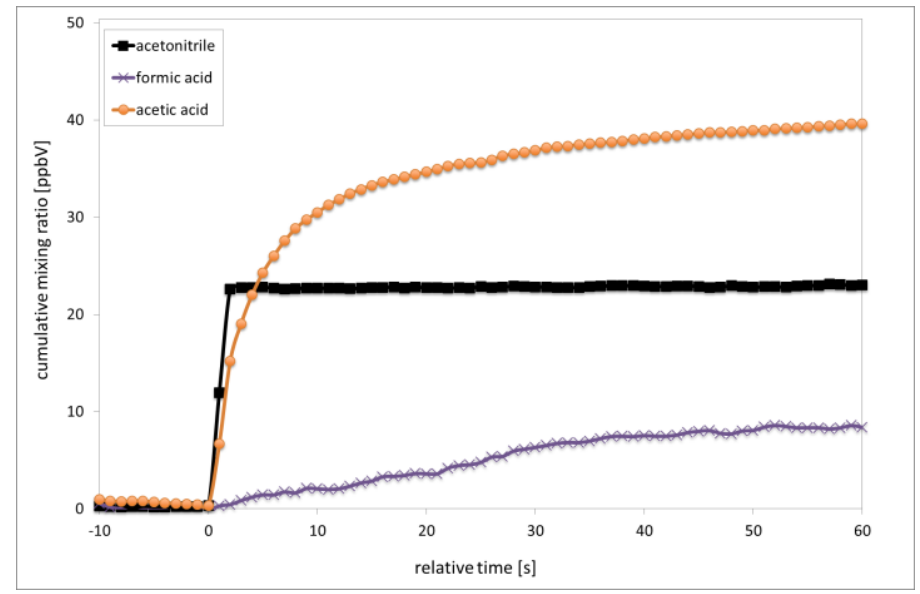

Figure S2. Cumulative mixing ratios of acetonitrile, formic acid and acetic acid as observed during the second fire overflight 


\section{Complete list of all detected signals}

Table S2. List of all ion signals detected in the plume, their elemental composition, associated emission factors (EF) and their standard deviation (SD), emission ratios (ER) and $\mathrm{SD}$, and $\mathrm{R}^{2}$ of the least-square regression analysis. For signal quantification see Section 2.2.

\begin{tabular}{|c|c|c|c|c|c|c|}
\hline $\mathbf{m} / \mathbf{z}$ & $\begin{array}{l}\text { elemental } \\
\text { composition }\end{array}$ & EF & SD & $\mathrm{ppbV/ppmV}$ & $\underset{n V}{\text { SD }}$ & $\mathbf{R}^{2}$ \\
\hline 30.00 & NO & 0.15 & 0.05 & 2.00 & 0.69 & 0.74 \\
\hline 31.02 & СН3О & 2.31 & 0.57 & 22.70 & 1.28 & 0.99 \\
\hline 33.03 & CH5O & 2.25 & 1.06 & 19.57 & 1.98 & 0.97 \\
\hline 41.04 & $\mathrm{C} 3 \mathrm{H} 5$ & 0.13 & 0.03 & 0.95 & 0.05 & 0.99 \\
\hline 42.03 & $\mathrm{C} 2 \mathrm{H} 4 \mathrm{~N}$ & 0.19 & 0.06 & 1.55 & 0.17 & 0.96 \\
\hline 43.02 & С2H3O & 0.24 & 0.08 & 1.85 & 0.10 & 0.99 \\
\hline 43.05 & $\mathrm{C} 3 \mathrm{H} 7$ & 0.64 & 0.25 & 4.51 & 0.22 & 0.99 \\
\hline 44.01 & $\mathrm{CH} 2 \mathrm{NO}$ & 0.07 & 0.02 & 0.62 & 0.20 & 0.77 \\
\hline 44.04 & $\mathrm{C} 2 \mathrm{H} 6 \mathrm{~N}$ & 0.02 & 0.00 & 0.11 & 0.01 & 0.95 \\
\hline 45.03 & С2H5O & 1.52 & 0.50 & 10.44 & 0.30 & 1.00 \\
\hline 45.99 & $\mathrm{NO} 2$ & 0.03 & 0.02 & 0.15 & 0.03 & 0.92 \\
\hline 47.02 & CH3O2 & $<0.13$ & 0.38 & $<1.38$ & 0.63 & 0.67 \\
\hline 54.03 & $\mathrm{C} 3 \mathrm{H} 4 \mathrm{~N}$ & 0.05 & 0.01 & 0.32 & 0.07 & 0.88 \\
\hline 57.04 & $\mathrm{C} 3 \mathrm{H} 5 \mathrm{O}$ & 0.17 & 0.04 & 0.94 & 0.05 & 0.99 \\
\hline 57.07 & C4H9 & 0.12 & 0.05 & 0.64 & 0.05 & 0.99 \\
\hline 59.05 & C3H7O & 0.83 & 0.31 & 4.15 & 0.11 & 1.00 \\
\hline 61.03 & C2H5O2 & 0.47 & 0.18 & 2.73 & 0.26 & 0.97 \\
\hline 69.03 & C4H5O & 0.25 & 0.12 & 1.02 & 0.11 & 0.97 \\
\hline 69.07 & С5H9 & 0.23 & 0.14 & 1.13 & 0.10 & 0.98 \\
\hline 71.05 & C4H7O & 0.33 & 0.12 & 1.44 & 0.05 & 1.00 \\
\hline 73.02 & C3H5O2 & 0.27 & 0.07 & 1.23 & 0.11 & 0.98 \\
\hline 73.07 & $\mathrm{C} 4 \mathrm{H} 9 \mathrm{O}$ & 0.21 & 0.08 & 0.87 & 0.04 & 0.99 \\
\hline 75.04 & $\mathrm{C} 3 \mathrm{H} 7 \mathrm{O} 2$ & 0.28 & 0.15 & 1.10 & 0.13 & 0.96 \\
\hline 79.05 & C6H7 & 0.40 & 0.15 & 1.42 & 0.04 & 1.00 \\
\hline 81.01 & С5H5O & 0.11 & 0.05 & 0.40 & 0.04 & 0.98 \\
\hline 83.05 & C5H7O & 0.18 & 0.09 & 0.66 & 0.07 & 0.97 \\
\hline 83.10 & C6H11 & 0.04 & 0.01 & 0.14 & 0.01 & 0.99 \\
\hline 84.07 & n.a. & 0.03 & 0.01 & 0.11 & 0.01 & 0.99 \\
\hline 84.99 & n.a. & 0.03 & 0.02 & 0.13 & 0.02 & 0.97 \\
\hline 85.04 & C4H5O2 & 0.39 & 0.12 & 1.48 & 0.09 & 0.99 \\
\hline 85.08 & С5H9O & 0.11 & 0.04 & 0.42 & 0.04 & 0.98 \\
\hline 87.04 & C4H7O2 & 0.44 & 0.18 & 1.62 & 0.09 & 0.99 \\
\hline 87.09 & $\mathrm{C} 5 \mathrm{H} 11 \mathrm{O}$ & 0.07 & 0.03 & 0.21 & 0.00 & 1.00 \\
\hline 89.04 & $\mathrm{C} 3 \mathrm{H} 5 \mathrm{O} 3$ & 0.03 & 0.01 & 0.08 & 0.00 & 0.99 \\
\hline 93.04 & C6H5O & 0.04 & 0.01 & 0.13 & 0.01 & 0.98 \\
\hline 93.07 & $\mathrm{C} 7 \mathrm{H} 9$ & 0.20 & 0.09 & 0.66 & 0.04 & 0.99 \\
\hline 95.05 & C6H7O & 0.26 & 0.11 & 0.95 & 0.06 & 0.99 \\
\hline 97.00 & n.a. & 0.18 & 0.09 & 0.60 & 0.04 & 0.99 \\
\hline 97.03 & C5H5O2 & 2.31 & 1.07 & 7.65 & 0.64 & 0.98 \\
\hline 99.02 & $\mathrm{C} 4 \mathrm{H} 3 \mathrm{O} 3$ & 0.14 & 0.04 & 0.51 & 0.10 & 0.90 \\
\hline 99.04 & $\mathrm{C} 5 \mathrm{H} 7 \mathrm{O} 2$ & 0.19 & 0.07 & 0.65 & 0.04 & 0.99 \\
\hline 101.03 & $\mathrm{C} 4 \mathrm{H} 5 \mathrm{O} 3$ & 0.03 & 0.03 & 0.16 & 0.00 & 0.98 \\
\hline 101.05 & $\mathrm{C} 5 \mathrm{H} 9 \mathrm{O} 2$ & 0.11 & 0.04 & 0.30 & 0.01 & 0.99 \\
\hline 103.05 & $\mathrm{C} 8 \mathrm{H} 7$ & 0.13 & 0.06 & 0.44 & 0.03 & 0.98 \\
\hline 105.07 & $\mathrm{C} 8 \mathrm{H} 9$ & 0.04 & 0.02 & 0.13 & 0.01 & 0.98 \\
\hline 107.09 & C8H11 & 0.19 & 0.07 & 0.51 & 0.02 & 1.00 \\
\hline 109.07 & C7H9O & 0.21 & 0.09 & 0.59 & 0.03 & 0.99 \\
\hline 111.05 & C6H7O2 & 0.39 & 0.21 & 1.25 & 0.14 & 0.96 \\
\hline 113.04 & C5H5O3 & 0.08 & 0.03 & 0.23 & 0.01 & 0.98 \\
\hline 117.05 & C5H9O3 & 0.07 & 0.02 & 0.13 & 0.02 & 0.94 \\
\hline 119.07 & C9H11 & 0.06 & 0.02 & 0.16 & 0.00 & 1.00 \\
\hline 121.10 & С9H13 & 0.10 & 0.03 & 0.25 & 0.01 & 0.99 \\
\hline 125.06 & $\mathrm{C} 7 \mathrm{H} 9 \mathrm{O} 2$ & 0.08 & 0.04 & 0.21 & 0.01 & 0.98 \\
\hline 127.05 & $\mathrm{C} 6 \mathrm{H} 7 \mathrm{O} 3$ & 0.08 & 0.04 & 0.21 & 0.02 & 0.99 \\
\hline 129.06 & С10H9 & 0.06 & 0.03 & 0.14 & 0.01 & 0.99 \\
\hline 133.07 & С9H9O & 0.05 & 0.02 & 0.12 & 0.01 & 0.99 \\
\hline 137.13 & $\mathrm{C} 10 \mathrm{H} 17$ & 0.19 & 0.12 & 0.44 & 0.06 & 0.94 \\
\hline
\end{tabular}

